\title{
Giant pulmonary chondroid hamartoma in a 74-year-old female patient. A case report and brief review of literature
}

\begin{abstract}
Pulmonary hamartomas are the most frequent benign tumors of the lung most commonly seen in men in the 5-6 $6^{\text {th }}$ decades of life. Usually smaller than $4 \mathrm{~cm}$ and asymptomatic they can occasionally present as large lesions. Herein we present a case of a very large chondroid hamartoma in a 74-year-old female patient along with a brief review of the literature.
\end{abstract}

\author{
Volume 6 Issue 3 - 2019

\begin{abstract}
Jan Pojda,' Sandra Hürlimann,' Alfred Leiser IInstitute of Pathology, Lucerne Cantonal Hospital, Switzerland ${ }^{2}$ Department of Thoracic Surgery, Lucerne Cantonal Hospital,
\end{abstract} \\ Switzerland
}

\begin{abstract}
Correspondence: Jan Pojda MD, Institute of Pathology, Lucerne Cantonal Hospital, Spitalstrasse, 6000 Lucerne, Switzerland,Tel+0041797707548, Email fortan1986@gmail.com
\end{abstract}

Received: June 03, 2019 | Published: June 10, 2019

\section{Introduction}

Pulmonary chondroid hamartomas, originally regarded as congenital developmental malformations, are now viewed as benign tumors derived from peribronchial mesenchyme. ${ }^{1}$ They are slow growing lesions most commonly seen in men in the sixth decade of life. Most are incidental findings and asymptomatic. Usually they are less than $4 \mathrm{~cm}$ in size, however rare cases of much larger lesions have also been reported. ${ }^{2,3,4}$ We report a case of incidental giant chondroid hamartoma of the right lower lobe of the lung in a 74-year-old female patient.

\section{Case report}

A 74-year-old female patient presented with symptoms of upper respiratory tract infection and mild progressive shortness of breath. She had a history of smoking, chronic obstructive pulmonary disease (COPD) GOLD stage 2 and arterial hypertension. A routine $\mathrm{X}$-ray examination of the chest revealed elevation of the right lung. Subsequent computed tomography showed a large heterogenous tumor measuring $14 \times 12 \times 11 \mathrm{~cm}$ above the right diaphragm, partly fatty and with calcifications. PET-tomography findings were similar and there was no contrast uptake. The radiological differential diagnoses included a pleural fibrous tumor owing to the proximity of the lesion the diaphragm as well as teratoma and low grade liposarcoma considering its partly fatty aspect. Due to the size of the lesion the possibility of a hamartoma was not considered at the time.

The tumor was removed by means of lateral thoracotomy. Intraoperatively it was found that the lesion had its point of origin in the right lower lobe of lung and had no direct connection to parietal pleura. A bundle of prominent blood vessels connecting the lesion with the lung and running towards the pulmonary hilus was ligated prior to its resection. A frozen section performed first raised the possibility of a benign chondroid hamartoma. The remaining lower lobe of the right lung was left intact with only the tumor being removed.

The tumorectomy specimen showed a tumor measuring $14.5 \times 12 \times 11 \mathrm{~cm}$ and with a weight of $1123 \mathrm{~g}$. The lesion was multilobulated on cross sections with some myxoid-appearing areas. Microscopic examination revealed heterogeneity of the tumor. Most of the lesion showed cartilaginous and fatty differentiation with extensive chondroid and chondro-myxoid areas. Also present were narrow slit-like spaces lined by simple columnar and pseudostratified ciliated epithelium. Focally adjacent there was a mild chronic inflammatory infiltrate as well as strands of smooth muscle and focal calcifications. The lesion was well circumscribed and attached to it was a small amount of unremarkable lung tissue. The diagnosis made was chondroid hamartoma or more specifically giant chondroid hamartoma owing to its unusually large size.

The patient made a quick and full recovery and was discharged from hospital 8 days following surgery. She also noted her shortness of breath had diminished following surgery. She has so far had an uneventful history (1 year follow-up) (Figure 1-4).

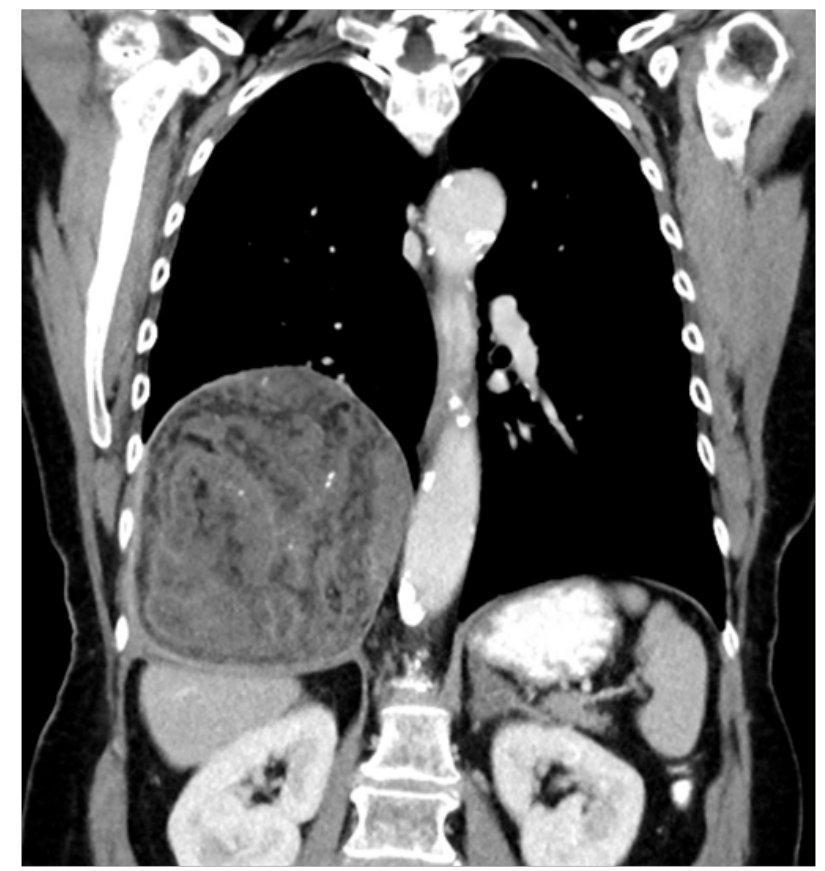

Figure I Radiologic imaging (CT) showing a large heterogeneous lesion in the lower right thoracic cavity. 


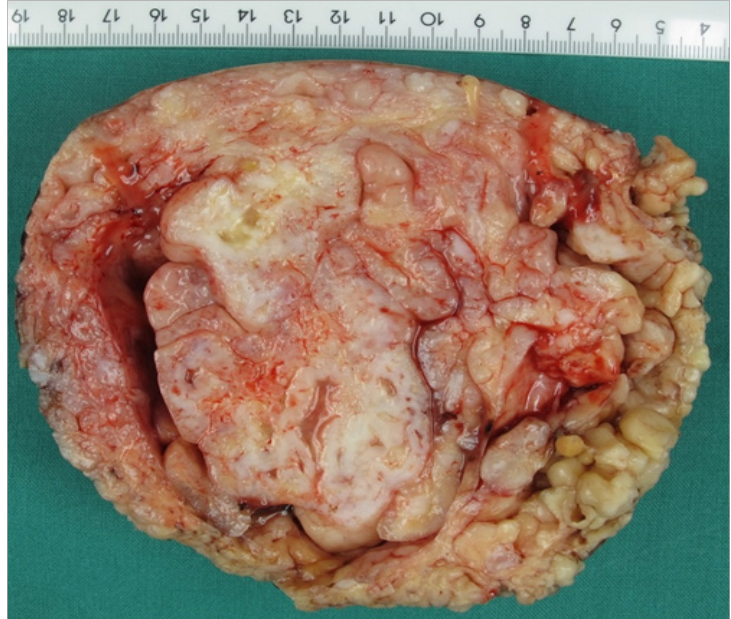

Figure 2 Gross image of the lesion showing well circumscribed, multilobulated tumour.

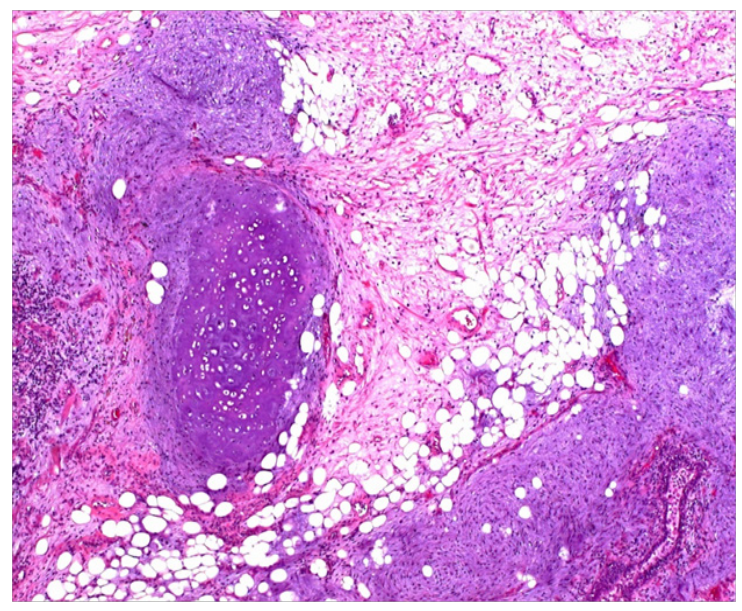

Figure 3 Microscopic image showing mature cartilaginous, chondromyxoid and fatty areas, hematoxylin and eosin staining.

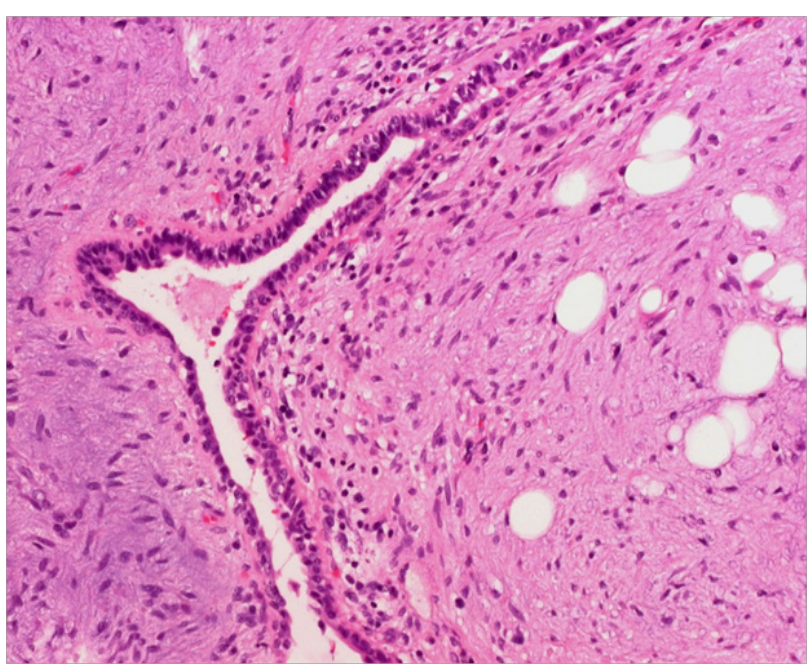

Figure 4 Microscopic image showing slit-like spaces lined by simple columnar and respiratory-type epithelium, hematoxylin and eosin staining.

\section{Discussion}

Pulmonary hamartomas (PHs), also called mixed mesenchymomas or chondromatous hamartomas, are the most common benign pulmonary tumors accounting for $6-8 \%$ of neoplasms of the lung. They are usually solitary, less than $4 \mathrm{~cm}$ in diameter and occur most frequently in the $5-6^{\text {th }}$ decades of life, more commonly in men with a male:female ratio of $4: 1$. They are thought to originate from the connective tissue underneath bronchial mucosa and are by definition composed of at least two mesenchymal elements. This differentiates them from monomorphic tumors such as chondroma. Most hamartomas show predominantly chondroid features (80\%) and are hence referred to as pulmonary chondroid hamartomas (PCHs). Typically alongside cartilage PHs also contain other mesenchymal elements such as adipose tissue, smooth muscle, fibrovascular tissue as well as slit-like spaces lined by entrapped respiratorytype epithelium. ${ }^{1,4,5,6,7}$ Based on location they can be divided into parenchymal $(90 \%)$ and endobronchial $(10 \%)$ lesions, the former usually being asymptomatic, the latter more frequently causing symptoms due to airway obstruction. Endobronchial lesions have also been found to contain somewhat less cartilage and epithelial clefts than intraparenchymal ones. ${ }^{5}$ Typically they are unencapsulated, lobulated lesions with connective tissue septa. ${ }^{2}$

Radiologically pulmonary hamartomas usually present as solitary pulmonary nodules (SPN) round and circumscribed with a smooth edge and a diameter of less than $2.5 \mathrm{~cm}$. As such they are described as having the features of so-called coin lesions. ${ }^{8,9}$ Aside from size, contour and stable size, the most important clue to the radiological diagnosis of hamartoma is the presence of fat, which is seen in as many as $50 \%$ of cases. ${ }^{10}$ Accordingly, a lesion with the appropriate size and demarcation showing focal fat attenuation of -40 to -120 Hounsfield units on computed tomography is in all likelihood a hamartoma. ${ }^{11}$ The much less likely differential diagnoses include lipoma and myelolipoma as well as metastatic liposarcoma or renal cell carcinoma. ${ }^{11}$ Lipomas are benign tumors consisting of mature adipose tissue, very rarely seen in the lung (or for that matter, other parenchymal organs). In the vast majority of cases they have an endobronchial location with peripheral tumors being exceedingly rare. ${ }^{12}$ Myelolipomas comprise mature adipose tissue admixed with haematopoietic elements and most commonly occur in the adrenal gland with intrathoracic cases mainly seen in the mediastinum and only exceptionally rare cases reported in the lung, some with an endobronchial, others with a parenchymal location. ${ }^{13,14}$

Another feature which can substantially aid in the diagnosis of PHs is the presence of calcifications which in hamartomas usually have the appearance of randomly distributed often overlapping rings, described as so-called popcorn calcifications. They are found in 5-50\% of hamartomas and correspond to the foci of chondroid calcification seen on histology.,15,16 As seen in our case, however, the radiological diagnosis can sometimes be challenging.

On magnetic resonance imaging pulmonary hamartomas are characterized by intermediate signaling on T1-weighted sequences, high signal intensity on $\mathrm{T} 2$-weighted sequences and no signal restriction on DWI sequences. ${ }^{16}$ MRI imaging can also reveal the presence of cleft structures in the lesion. ${ }^{17}$ In addition, chemical shift MRI has been proposed as an additional tool to aid in the detection of fat in hamartomas. ${ }^{18}$

PHs are benign slow growing lesions with an expansion rate of $3.2+/-2.6 \mathrm{~mm}$ per year as reported by Hansen et al. ${ }^{2}$ which is why 
some authors recommend surveillance rather than operative treatment if there is no doubt regarding the diagnosis. They recommend surgery only in cases with pulmonary symptoms or in cases of enlarging lesions in young and middle-aged patients. ${ }^{2,19}$ Complications of hamartomas are rarely seen with pneumothorax reported as a complication in two cases of giant hamartoma with a cystic aspect. ${ }^{20,21}$

Rarely PHs can reach considerable dimensions with Zong et al. ${ }^{21}$ reporting a lesion $30 \mathrm{~cm}$ in diameter in $2014 .{ }^{23}$ Due to their size these large tumors are referred to as giant pulmonary hamartomas. Giant PHs, while more common in adults, have also been observed in children and have been described to possess a somewhat cystic aspect in some reported pediatric cases. ${ }^{9,24,25}$ Of note is a case of giant PH measuring $18 \mathrm{~cm}$ in an 11 year old boy described by Ozbudak et al. ${ }^{24}$ The main differential diagnosis in pediatric cases of $\mathrm{PHs}$ is that of a malignant pleuropulmonary blastoma. ${ }^{3}$ Very rarely PCHs hamartomas present as multiple tumors. ${ }^{26}$ In these cases it is important to discriminate between true pulmonary chondroid hamartomas and multiple pulmonary chondromas the latter being a different entity usually seen in young females as one of the manifestations of Carney's Triad, the other two being GISTs and extra-adrenal Paragangliomas. ${ }^{27,28} \mathrm{~A}$ hamartomatous entity presenting with multiple pulmonary lesions is the multiple leiomyomatous hamartoma, which occurs predominantly in women and have therefore by some been considered to be pulmonary metastases of benign leiomyomas, a view contradicted by very rare reports of similar lesions in men..$^{29,30,31}$

Pulmonary chondroid hamartomas have been found to frequently harbor certain genetic alterations involving genes encoding highmobility group or HMG proteins. ${ }^{23}$ In a study of 191 cases Kazmierczak et al..$^{31}$ found locus 12q14-15 encoding HMGI-C to be most commonly involved with a frequent translocation of $\mathrm{t}(12 ; 14)(\mathrm{q} 15 ; \mathrm{q} 24)$. The locus $6 \mathrm{p} 21$ encoding HMGI(Y) was the second most commonly involved with $\mathrm{t}(6 ; 14)(\mathrm{p} 21.3 ; \mathrm{q} 24)$ being a frequent translocation. ${ }^{32}$ It is of note that similar genetic abnormalities have been described in other benign mesenchymal tumors such as lipomas, leiomyomas, fibroadenomas, pleomorphic adenomas as well as endometrial polyps. ${ }^{33,34}$ This supports the view that pulmonary chondroid hamartomas represent true neoplasms rather than actual hamartomas or developmental anomalies. ${ }^{26}$

\section{Conclusion}

The diagnosis of pulmonary hamartoma can often be made with a high level of certainty on the basis of radiology alone. Occasionally, cases with a less typical radiological presentation can pose a diagnostic challenge as in our case which had the appearance of a large pleuraassociated tumor rather than a solitary pulmonary nodule. This shows that despite being the most common benign pulmonary tumors, chondroid hamartomas can in rare cases present as a diagnostic conundrum.

\section{Acknowledgements}

Dr. Christian Blumer, CA Institut für Radiologie, Zuger Kantonsspital.

\section{Conflicts of interest}

Author declares that there is no conflict of interest.

\section{References}

1. Hasleton P, Flieder D. Spencer's Pathology of the Lung. $6^{\text {th }}$ ed. Cambridge University Press;2013:224-1227.

2. Hansen CP, Holtveg H, Francis D, at al. Pulmonary hamartoma. J Thorac Cardiovasc Surg. 1992;104(3):674-678.

3. Ganti S, Milton R, Davidson L, et al. Giant pulmonary hamartoma. Journal of Cardiothoracic Surgery. 2006;1:19.

4. Travis W, Brambilla E, Burke A, et al. WHO Classification of tumors of the Lung, Pleura, Thymus and Heart. IARC. 2015:116-117.

5. Gjevre JA, Myers JL, Prakash UB. Pulmonary hamartomas - Mayo. Mayo Clin Proc. 1996;71(1):14-20.

6. Tomashefski JF Jr. Benign endobronchial mesenchymal tumors: their relationship to parenchymal pulmonary hamartomas. Am J Surg Pathol. 1982;6(6):531-40.

7. Hutter J, Reich-Weinberger S, Hutarew G, et al. Giant pulmonary hamartoma - a rare presentation of a common tumor. Ann Thorac Surg. 2006;82(2):e5-7.

8. Ray JF, Lawton BR, Magnin GE, et al. The coin lesion story: update twenty years' experience with early thoracotomy for 179 suspected malignant coin lesions. Chest. 1976;79:332-336.

9. Saadi MM, Barakeh DH, Husain S, et al. Large multicystic pulmonary chondroid hamartoma in a child presenting as pneumothorax. Saudi Medical Journal. 2015;36(4):487-489.

10. Gaerte SC, Meyer CA, Winer-Muram HT, et al. Fat-containing lesions of the chest. Radiographics. 2002;22:S61-78.

11. Collins J, Stern S. Chest Radiology: The Essentials. 3rd Edition. Lippincott Williams and Wilkins; 2014.

12. Civi K, Ciftçi E, Gürlek Olgun E, et al. Peripheral intrapulmonary lipoma: a case report and review of the literature. Tuberk Toraks. 2006;54(4):374377.

13. Sato K, Ueda Y, Katsuda S, et al. Myelolipoma of the lung: a case report and brief review. J Clin Pathol. 2007;60(6):728-730.

14. Mašić S, Vučić M, Seiwerth S. Pulmonary myelolipoma containing osseous tissue: An unexpected finding at autopsy. Respir Med Case Rep. 2017;22:254-256.

15. Gleeson T, Thiessen R, Hannigan A, et al. Pulmonary hamartomas: CT pixel analysis for fat attenuation using radiologic-pathologic correlation. J Med Imaging Radiat Oncol. 2013;57(5):534-43.

16. Siegelman SS, Khouri NF, Scott WW Jr, et al. Pulmonary hamartoma: CT findings. Radiology. 1986;160(2):313-317.

17. Park KY, Kim SJ, Noh TW, et al. Diagnostic efficacy and characteristic feature of MRI in pulmonary hamartoma: comparison with CT, specimen MRI, and pathology. J Comput Assist Tomogr. 2008;32(6):919-925.

18. Hochhegger B, Marchiori E, Quinto dos Reis D, et al. Chemical-Shift MRI of Pulmonary Hamartomas: Initial Experience Using a Modified Technique to Assess Nodule Fat. Am J Roentgenol. 2012;199(3):W331W334.

19. Elsayed H, Abdel Hady SM, Elbastawisy SE. Is resection necessary in biopsy-proven asymptomatic pulmonary hamartomas? Interact Cardiovasc Thorac Surg. 2015;21(6):773-776.

20. Stella F, Davoli F, Brandolini J, et al. A rare case of giant cystic chondroid hamartoma of the lung presenting with left side pneumothorax. Minerva Chir. 2009;64(1):117-119. 
21. Zong Y, Sun X. A Rare Case of Giant Pulmonary Hamartoma. J Pulm Respir Med. 2014;4:205.

22. Fletcher JA, Pinkus GS, Donovan JK, et al. Clonal rearrangement of chromosome band 6 p21 in the mesenchymal component of pulmonary chondroid hamartoma. Cancer Res. 1992;52:6224-6228.

23. Ozbudak IH, Dertsiz L, Bassorgun CI, et al. Giant cystic chondroid hamartoma of the lung. J Pediatr Surg. 2008;43:1909-1911.

24. Kim GY, Han J, Kim DH, et al. Giant Cystic Chondroid Hamartoma. Journal of Korean Medical Science. 2005;20(3):509-511.

25. Fan M, Lin Y, Liu L. Multiple pulmonary chondroid hamartoma. J Thorac Oncol. 2014;9(7):1053-1054.

26. Rodriguez FJ, Aubry MC, Tazelaar HD, et al. Pulmonary chondroma: a tumor associated with Carney triad and different from pulmonary hamartoma. Am J Surg Pathol. 2007;31(12):1844-1853.

27. Bateson E. Cartilage-containing tumours of the lung. Relationship between the purely cartilaginous type (chondroma) and the mixed type (so-called hamartoma): an unusual case of multiple tumours. Thorax. $1967 ; 22: 256$

28. Nistal M, Hardisson D, Riestra ML. Multiple pulmonary leiomyomatous hamartomas associated with a bronchogenic cyst in a man. Archives of Pathology \& Laboratory Medicine. 2003;127(4):e194-e196.
29. Nili M, Vidne BA, Avidor I et al. Multiple pulmonary hamartomas; a case report and review of the literature. Scand J Thorac Cardiovasc Surg. 1979;13(2):157-160.

30. Logan WD, Rohde FC, Abbott OA, et al. Multiple pulmonary fibroleiomyomatous hamartomas: report of a case and review of the literature. Am Rev Res Dis. 1965;91:101-103.

31. Kazmierczak B, Meyer-Bolte K, Tran KH, et al. A high frequency of tumors with rearrangements of genes of the HMGI(Y) family in a series of 191 pulmonary chondroid hamartomas. Genes Chromosomes Cancer. 1999;26(2):125-133.

32. Hess JL. Chromosomal translocations in benign tumors: the HMGI proteins. Am J Clin Pathol. 1998;109(3):251-261.

33. Tallini G, Vanni R, Manfioletti G, et al. HMGI-C and HMGI(Y) immunoreactivity correlates with cytogenetic abnormalities in lipomas, pulmonary chondroid hamartomas, endometrial polyps, and uterine leiomyomas and is compatible with rearrangement of the HMGI-C and HMGI(Y) genes. Lab Invest. 2000;80(3):359-369.

34. Johansson M, Dietrich C, Mandahl N, et al. Recombinations of chromosomal bands $6 \mathrm{p} 21$ and $14 \mathrm{q} 24$ characterise pulmonary hamartomas. Br J Cancer. 1993;67(6):1236-1241. 